Capítulo 9

\title{
LAS DINÁMICAS DE RESISTENCIA DE LA POBLACIÓN DE ARACATACA AL TRANSPORTE FÉRREO DE CARBÓN EN MAGDALENA, COLOMBIA, PERIODO 2010-2016
}

\author{
Hernando Parra ${ }^{1}$ \\ Kendy Paola Tapia Díaz ${ }^{2}$ \\ Oscar Iván Arias Muñoz
}

\section{Resumen}

Este artículo tiene como propósito principal referir los elementos centrales tratados en la investigación sobre Las Dinámicas de resistencia de la Población de Aracataca al Transporte Férreo de Carbón en el Departamento del Magdalena Durante el Periodo 20102016. Dichos elementos giran en torno a tres temas de discusión: en primer lugar, sobre el Modelo Extractivista implementado en Colombia; en segundo lugar, sobre los conflictos socio-ambientales causados por la actividad minera en el caribe colombiano; en tercer lugar, la naturaleza del movimiento de resistencia social de la población de Aracataca al paso de tren carbonero por el casco urbano del municipio.

1 Sociólogo, Magíster en Desarrollo Social, profesor investigador del grupo Goffman, programa de Sociología de la Universidad del Atlántico

2 Magíster en Socióloga de la Universidad del Atlántico

3 Magíster en Sociólogo de la Universidad del Atlántico 
Palabras clave: Extractivismo, conflicto, Resistencia, Paraestado.

\begin{abstract}
The main purpose of this article is to refer to the central elements dealt with in the research on The Dynamics of Resistance of the Population of Aracataca to Coal Ferrous Transport in the Department of Magdalena During the 2010-2016 Period. These elements revolve around three topics of discussion: first, on the Extractivist Model implemented in Colombia; second, on the socio-environmental conflicts caused by mining activity in the Colombian Caribbean; third, on the nature of the social resistance movement of the population of Aracataca to the passage of coal trains through the urban area of the municipality.
\end{abstract}

Keywords: Extractivism, conflict, Resistance, Parastate

\title{
Introducción
}

El estudio sobre "Las Dinámicas de Resistencia de la Población de Aracataca al Transporte Férreo de Carbón en el Departamento del Magdalena Durante el Periodo 2012-2016" asumió como propósito principal de investigación el de describir la resistencia de la población de Aracataca al paso del transporte férreo de carbón por su municipio, cuya justificación de resistencia son las consecuencias ambientales, económicas, sociales y de salud que afecta a sus habitantes. El estudio aborda la explicación causal de la problemática a partir de una exposición sobre "la lógica del extractivismo", esto es, que ésta se define "como una forma de organización de la economía del país basada en una alta dependencia de la extracción intensiva (en grandes volúmenes) de recursos naturales, con muy bajo procesamiento y destino para la exportación" (p. 12). En este sentido se afirma que Colombia siendo un país con grandes recursos naturales ha asumido, desde los años ochenta del Siglo XX, una política económica extractivista, basada en los recursos minero-energéticos. De tal manera, que los últimos gobiernos han considerado a este sector como el motor del crecimiento económico del país, tomando como único referente la evolución del PIB.

Entonces, efectivamente, los gobiernos, desde una "teoría globalizada del desarrollo (...) El desarrollo es comprendido en términos generales como crecimiento económico, visión que se convierte en pilar de la vida social 
moderna, pero que desconoce los límites físicos, biológicos y geológicos del planeta". (Garavito 2016, p. 182) han orientado los planes nacionales de desarrollo con un interés puramente económico, siendo así, por ejemplo, que en "El Plan Nacional de Desarrollo 2010-2014 (Prosperidad Democrática)" la locomotora minero-energética lidera la propuesta del crecimiento económico del país. Sin embargo, se argumenta que este modelo minero-energético conlleva una gran paradoja en Colombia, pues, por una parte, se muestra como el eje dinamizador de la economía del país, a través de su participación en el PIB y en las ventas de exportación (Llinás, 2010), pero, que, por la otra, se constituye en el factor más significativo en la generación de conflictos, pobreza y deterioro del medio ambiente en las regiones donde realiza su actividad (Garay, 2013).

Ahora bien, se señala que dicha actividad extractivista en Colombia es identificada por el profesor Guillermo Rudas de la siguiente manera:

La producción tanto de hidrocarburos como de los principales productos mineros del país destinados a la exportación (carbón, oro y níquel) se encuentra altamente concentrada en 7 de los 33 departamentos... y que... el carbón se origina mayoritariamente en los departamentos del Cesar y La Guajira, con $48 \%$ y $42 \%$, respectivamente, de la producción nacional...y que... la totalidad del ferroníquel seproduce únicamente en el departamento de Córdoba (...) (2013, pp. 28-84)

Es decir, que "alrededor del 90\% del carbón que se ha producido en los últimos años en Colombia se origina en los departamentos de Cesar y Guajira, siendo éste exclusivamente destinado a la exportación" (p. 46). En este contexto, los estudios sobre la actividad minera en la Región Caribe han orientado su trabajo a partir de tres momentos importantes de la ruta del carbón: explotación, transporte y embarque. Donde, por supuesto, se considera que en cada momento las consecuencias ambientales, económicas, sociales y de salud tienen sus especificidades. Por ello, a partir de lo arriba señalado, la investigación asumió el momento del transporte de carbón. Las dos modalidades de transporte de carbón han sido por carretera a través de camiones y por vía férrea a través de trenes. Sin embargo, en los últimos años la primera modalidad ha ido perdiendo importancia, por ello la investigación se centró en la segunda modalidad. Considerando que las especificidades de los impactos ambientales y sociales están presentes en 
él, pues es conocido que "el transporte de carbón emite a su paso por las comunidades grandes cantidades de polvillo, contaminando la atmósfera y la calidad del aire". Dicha actividad transportadora se realiza a través de La Concesión del Atlántico, que es una red férrea exclusiva para el transporte de carbón desde Chiriguaná (Cesar) hasta Santa Marta (Magdalena), la cual está al servicio de las multinacionales explotadoras de carbón, tales como: Drumond, Prodeco, Glencore, Vale Do Río y Carbones del Cesar.

Por su parte, la investigación centró su mirada en la población de Aracataca, puesto que ella ha dado las muestras de resistencia al paso del tren carbonífero por su municipio de manera muy significativa, pues allí se organizaron y movilizaron las instituciones públicas, las privadas y las comunidades. Efectivamente, la población se organizó en un comité pro-defensa al paso del tren carbonero por el municipio, cuyas acciones proporcionaron unidad participativa a los manifestantes por más de diez años, logrando con su movilización conectarse con el sentido de resistencia de un movimiento social más general al modelo extractivista en Colombia y América Latina. Sobre las especificidades de las dinámicas de resistencia de la población de Aracataca al paso del tren carbonero por su municipio, la investigación encontró que, efectivamente, el paso del tren afecta la salud, la calidad de vida, y la economía de sus habitantes a través del polvillo del carbón, de la construcción de una segunda línea férrea, de la construcción de una valla (Muro) divisoria del municipio, entre otros, lo cual conllevó a que sus habitantes realizaran asambleas, plantones, movilizaciones y a crearan formas organizativas como una Veeduría Ciudadana Pluralista ProDefensa de Aracataca, en adelante VEECPRODEA.

En síntesis, la investigación pudo observar que las dinámicas de resistencia que ha llevado a cabo la población de Aracataca se inscriben en la lógica de grandes movilizaciones nacionales que luchan por la defensa del medioambiente, los territorios y la salud humana, tales como "el movimiento social Defensores del Territorio", encabezado por el profesor Miller Dussan en su lucha activa en contra de la construcción de la represa hidroeléctrica del Quimbo en el Departamento del Huila. 


\section{Metodología}

La metodología utilizada en la investigación se centró en la Investigación Total de Hugo Cerda, la cual permitió triangular información de tipo cualitativo, cuantitativo y la de los expertos. En ese sentido, se trianguló la información de los habitantes de los barrios más significativos en los eventos de resistencia, tales como Cataquita, Macondo, el Suiche, Siete de Agosto, Siete de Abril, Camellón, El Pradito, El Centro, El Carmen, Boston y San José, con los cuales se realizaron dos grupos focales y once entrevistas semi-estructuradas, tres Historias de Vida a líderes significativos de la movilización; dicha información se trianguló con la información de la parte institucional del municipio, ya que se realizaron ocho entrevistas semi-estructuradas a representantes de la Institucionalidad, tales como al Alcalde y a Concejales del municipio; también se trianguló con información de expertos de la región, tales como la del investigador Fabio Silva Vallejo.

\section{Principales hallazgos}

Los primeros hallazgos de la investigación se relacionan con las motivaciones de la resistencia de la población de Aracataca al paso del tren carbonero por el centro del casco urbano del municipio: un tren de ciento veinte vagones que atraviesa la población unas cuarenta y ocho veces al día, dividiéndola en dos, porque la línea férrea al estar ubicada en el centro del municipio divide del costado oriental 16 barrios en donde se encuentra la Alcaldía, el Concejo Municipal, su zona comercial y algunas instituciones educativas y del lado occidental se encuentran 18 barrios, el hospital y algunas instituciones educativas. Las afectaciones a la población se evidenciaron así:

Según un habitante del barrio el Suiche, el paso del tren es muy peligroso para todos estos pueblos del Magdalena, Algarrobo, Fundación, Aracataca, porque el polvillo contamina desde el centro de la carrilera, 3 kilómetros a un lado y 3 kilómetros al otro, el radio de acción es de 3 kilómetros, uno que está cerca percibe más rápido, en Aracataca hay personas que creen que porque no viven a la orilla de la vía no reciben la contaminación pero si la reciben tanto como uno, en el día quizás por el movimiento uno no inhala tanto, pero en la noche 
al dormir recibimos todo eso, por eso, hemos optado por tener aire acondicionado y tapar bien las cosas porque anteriormente teníamos nada más el abanico y al analizar nos dábamos cuenta que estaba forrado de negro. En el verano el eternit acumula todo el polvillo y cuando llueve las primeras gotas que caen son negras. En las hojas de los árboles también se pega porque en la mañana con el rocío las hojas se humedecen y con el pasar de los trenes el polvillo se les pega, tú puedes ver a las horillas de la línea como las hojas se ven negras del polvillo que se les pega.

Un habitante del barrio Camellón 20 de julio informó que el tren divide completamente el municipio y poseen una sola línea, el único lugar del mundo donde una ambulancia se detiene con un herido abordo para que el tren pase es en Aracataca, porque en otros lugares los automóviles le tienen que dar el paso a la ambulancia. Entonces, es ellos nosotros muy importante levantar la voz, son un pueblo que lucha por la vida de todos sus habitantes. El concejal Alejandro Mora manifiesta que a los concejos les corresponde velar por el ambiente sano de las comunidades y siempre han manifestado que los trenes carboneros de Fenoco contaminan el ambiente al pasar por el centro del casco urbano municipal de Aracataca. Situación que se demuestra no porque tuvieron temerarios de sus acusaciones, sino que se ha demostrado con el tiempo que es contaminante, no solo en el aire, sino también visual, auditivo e impide la libre movilidad de la población; dividiendo no solo geográficamente, sino también el componente Institucional.

Un habitante del barrio Cataquita dice que hay muchas casas que están rajadas, y cree que en el corredor férreo no hay una casa que no esté rajada. Otro habitante del barrio El suiche, comenta que siempre están en vía de defender primero que todo la salud, no solo de ellos sino también de los familiares y paisanos y generaciones que vienen detrás porque en realidad el carbón es nocivo para la salud, parecido al asbesto, entra en los pulmones a los bronquios y jamás sale, los residuos que bota el tren a su paso, con todos los cuidados que tienen para no contaminar entra en los pulmones y no sale más, tanto así que los ingenieros de minas que trabajan con carbón y son pensionados a los 8 años y las personas también las pensionan o las botan a temprana edad porque son desechables. En 
segundo lugar, los hallazgos se relacionan con las dinámicas de resistencia de la población de Aracataca al paso del tren carbonero por el casco urbano del municipio. Efectivamente, la población de Aracataca, con base en la experiencia de resistencia de los movimientos sociales al modelo extractivista en América Latina, logra identificar las especificidades de la resistencia a las consecuencias ambientales y sociales de la ruta del carbón en el caribe colombiano, asumiendo las que tienen que ver con el transporte férreo de carbón.

En ese sentido, la primera estrategia de resistencia fue la de organizarse, la cual se concretó a través de VEECPRODEA, pues consideraron que desde este marco de legalidad podía mantener la unidad para informar, vigilar y proponer. Además, con el fin de estructurar el movimiento de resistencia desde la misma base se optó por las asambleas comunitarias, lideradas por las juntas de acción comunal. Una segunda estrategia fue la construcción de un discurso de resistencia de la población para la movilización, con el fin de contrarrestar el discurso oficial de las multinacionales. Dicho discurso de resistencia se construyó y expresó a través de asambleas, plantones, carteles, pasacalles, consignas, canciones, demandas, derechos de petición, etc. La tercera estrategia de resistencia se fundamentó en la participación directa, que los pobladores barriales de Aracataca crearon a través de nodos de oralidad interna, los cuales permitieron el empoderamiento comunitario para su participación activa en el movimiento general de resistencia de la población, logrando en ese proceso sobreponerse al miedo a la judicialización generado por las multinacionales a través del poder local.

Otro hallazgo importante está relacionado con la caracterización que el estudio hace de la resistencia de la población de Aracataca al paso del tren carbonero por casco urbano del municipio, cuando lo define como un movimiento de resistencia al modelo extractivista, cuyas características están emparentadas con "la lógica de las grandes movilizaciones ambientalistas que se presentan en Latinoamérica", sin importar que sus especificidades sean "movilizaciones que tienden a ser más locales y puntuales, siempre orientados a favorecer a los sectores excluidos, a denunciar los impactos que sobre los recursos naturales y los sectores sociales tiene la instrumentación de algunos megaproyectos" (Arias y Tapia, 2018, pág. 69). 


\section{Discusión}

Un primer tema de discusión propuesto por la investigación está relacionado con el modelo extractivista implementado en Colombia como política económica por los gobiernos a partir del 2002, reivindicando a la minería por su "utilidad pública e interés social" para el país. Efectivamente, con esa mirada puesta solamente en el crecimiento económico, se observó el incremento del PIB a partir del aporte de la actividad minera durante el periodo 2000-2011: que "pasó de representar el 1.8\% al 2.3\% del PIB, incrementando su valor total de un 5.2 a un 10.3 billones de pesos, así mismo, las exportaciones de minerales duplicaron sobradamente su participación en las ventas externas, pasando de $9.8 \%$ a $21.3 \%$ en el mismo intervalo" (Cansino et al, 2014, p. 22).

Sin embargo, sin desconocer la contribución de la actividad minera al crecimiento económico y a las exportaciones, el periodo también ha mostrado claros procesos de "desindustrialización y una desagriculturación relativa en el país cuasi-estructural en los últimos años” (Espitia, 2014, p. 435), como producto de las relaciones que establece el sector minero con los otros sectores de la economía nacional. Efectivamente, el modelo extractivista ha prosperado con resultados muy contradictorios, pues:

El Estado mantuvo su condición de propietario del subsuelo, pero en la práctica las reservas descubiertas y explotadas fueron privatizadas a cambio de regalías que no compensan debidamente su agotamiento, ni han generado la riqueza esperada para el país, ni han contribuido al mejoramiento de la calidad de vida de las poblaciones afectadas. Antes, por el contrario, es evidente el deterioro del bienestar en algunas regiones del país, donde la minería es la actividad predominante, como se ha ilustrado en los diferentes estudios mineros publicados por la CGR (Pardo, 2014, p. 34)

La causa de la situación arriba señalada, según Espitia (2014, p. 410), es porque el modelo extractivista es de enclave, es decir, cuando un modelo económico permite que las compañías extranjeras se localicen "en un determinado país para extraer algún recurso no renovable para la exportación con muy poca integración con el mercado interno". 
Efectivamente, la economía de enclave de la minería se ve reflejada en la dinámica del flujo de divisas para el país, pues:

El actual sistema cambiario permite que el flujo de recursos de las compañías extranjeras mineras se limiten al monto base necesario para financiar los gastos laborales, en lo fundamental, ya que la maquinaria y equipo se adquiere en el exterior y los servicios se contratan y pagan en el exterior, mientras que la producción exportada (que es casi su totalidad) sale del país en especie sin que exista la obligación de que estos recursos monetizados, una vez sean vendidos, ingrese al país (Espitia, 2014, p. 410)

Por otra parte, cuando los estudios realizan un balance de la actividad minera, encuentran un "desbalance integral" que se profundiza día a día al momento de tomar en consideración "la gravedad y perdurabilidad de los múltiples y diversos impactos en los sistemas ambiental, social, cultural, territorial y de salud pública asociados con la explotación minera en el país" (Pardo, 2014, p. 35). Un segundo tema de discusión propuesto en la investigación de Arias y Tapia (2018) está relacionado con los conflictos socio-ambientales que ha generado el modelo extractivista, especialmente con los que tienen que ver con el carbón en tanto que es en la región caribe donde se ubica la mayor producción exportadora de este mineral y, en segundo lugar, con los surgidos por su transporte férreo que produce profundas afectaciones ambientales y sociales a todo lo largo de su recorrido, sabiendo que:

Durante el proceso de remoción del carbón del subsuelo, así como en el transporte y embarque, diversos fenómenos físicos permiten que el mineral forme pequeñas partículas, cuyo tamaño es muy variado, abarcando diámetro desde menos de una micra hasta varios milímetros, comúnmente conocidas como "polvillo de carbón" (Karkhanis y Joshi, 2013. Citados por Olivero, 2013, p. 239)

Es claro, entonces, que el transporte férreo de carbón en su recorrido genera impactos negativos en el ambiente, en la salud humana y en la infraestructura. Esto es, que los contaminantes se transportan, se diluyen y producen cambios físicos y químicos, afectando el aíre, el agua, la 
tierra, la vida silvestre y la vida humana. En este sentido, las principales afectaciones, registradas por la investigación, que reclama la población de Aracataca al paso del tren carbonero por el casco urbano de su municipio están relacionadas con el manejo y control de la empresa Fenoco con el carbón transportado, pues los vagones pasan sin las respectivas carpas que cubren el carbón, con la salud respiratoria de los habitantes por el "polvillo del carbón", con la salud auditiva de los habitantes, por el paso pitando de 3 locomotoras con 150 vagones cada 20 minutos durante las 24 horas del día, con la calidad de vida de los habitantes, pues la línea férrea pasa por el centro del municipio, perturbando las actividades educativas, hospitalarias, económicas y administrativas en el municipio, con la infraestructura habitacional y de comercio por el agrietamiento de paredes y pisos en las casas, además, con la propuesta de creación de una segunda línea férrea y la construcción de una valla protectora, que contribuiría más con la división del municipio.

Ante las reclamaciones de la población de Aracataca al Estado por las afectaciones, arriba señaladas, éste responde con una relativa pasividad, dado su marcado interés por el crecimiento económico a partir de la actividad minera, pero, que a su vez, responde con mano dura a cualquier perturbación del proceso, es decir, que muestra claramente su compromiso con el desarrollo de la minería de grandes proyectos de economía de enclave, donde no hay consideración por los diversos impactos ambientales y sociales. Por su parte, las empresas multinacionales responden, amparados en la excesiva tolerancia del Estado, enfocadas solamente en la argumentación economicista de "los mayores ingresos por concepto de la inversión extranjera, impuestos y regalías y el crecimiento de la producción bruta y las exportaciones asociadas a la explotación minera" (Pardo, 2014, p. 46). Esto es, que "el papel del Estado -desmedidamente en cabeza del ejecutivo nacional- se redujo a concesionar sin mayores requisitos, a regular y a fiscalizar el desarrollo de la minería, función que tampoco ejecutó con diligencia" (Pardo, 2014, p. 36).

Con todo ello se devela la conflictividad inter-institucional entre el poder local y el poder nacional, puesto que la postura de la población de Aracataca es apoyada por la Alcaldía y el Concejo Municipal, entre otros, pero reprimida por el Estado, donde, entonces, claramente se expresa un conflicto social a partir de la comprensión de la jerarquía de derechos 
previsto en el ordenamiento jurídico colombiano.Al respecto, Negrete (2013), recuerda que en la Constitución Política de 1991 están consagrados los derechos fundamentales que el Estado debe proteger con prioridad, los cuales han sido jerarquizados por algunos juristas como de primera, segunda y tercera generación:

Los Derechos Humanos de primera generación hacen referencia a los derechos civiles: derecho a la vida, la libertad, la integridad física, libertad de expresión, a un juicio justo, de religión y políticos (el sufragio). Los de segunda generación se refieren a los derechos económicos, sociales y culturales; y los de tercera generación al conjunto de derechos producto de acuerdos de la comunidad internacional, como los de demanda de un medio ambiente sano y libre de problemas. Los Derechos Humanos de tercera generación, surgidos en la doctrina en los años ochenta, se vinculan con la solidaridad. Los unifica su incidencia en la vida de todos, a escala universal, por lo que precisan para su realización una serie de esfuerzos y cooperaciones en un nivel planetario y universal. Dentro de estos se encuentran el derecho a la autodeterminación, a la identidad nacional y cultural, a la paz, a la coexistencia pacífica, a la solución de los problemas alimenticios, demográficos, educativos y ecológicos, a un medio ambiente sano y al desarrollo que permita una vida digna.

Además, que en ese sentido la Corte Constitucional, a través de la sentencia C-595/10, se pronunció frente al derecho a un ambiente sano: El interés superior del ambiente sano para la subsistencia de la humanidad. Los deberes constitucionales y los principios que lo fundamentan:

La persona y el entorno ecológico. Colombia es una Estado personalista fundado en el respeto de la dignidad humana consignado en el artículo $1^{\circ}$ superior. La Constitución reconoce la primacía de los derechos inalienables del ser humano, en el artículo $5^{\circ}$ superior. Se ha de proteger, entonces, el derecho a la vida, esto es, que la persona exista, además de garantizar cierta calidad de subsistencia. Sobre la Dignidad humana, artículo $1^{\circ}$ superior.

La persona es el sujeto, la razón de ser y fin último del poder político por lo que se constituye en norte inalterable en el ejercicio del poder y su relación con la sociedad. Es a partir del respeto por la vida humana 
que adquiere sentido el desarrollo de la comunidad y el funcionamiento del Estado. La Constitución muestra igualmente la relevancia que toma el medio ambiente como bien a proteger por sí mismo y su relación estrecha con los seres que habitan la tierra. La conservación y la perpetuidad de la humanidad dependen del respeto incondicional al entorno ecológico, de la defensa a ultranza del medio ambiente sano, en tanto factor insustituible que le permite existir y garantizar una existencia y vida plena. Desconocer la importancia que tiene el medio ambiente sano para la humanidad es renunciar a la vida misma, a la supervivencia presente y futura de las generaciones.

En el mundo contemporáneo, la preocupación ambientalista viene a tomar influencia decisiva solamente cuando resulta incuestionable que el desarrollo incontrolado y la explotación sin límites de los recursos naturales logran suponer su esquilmación definitiva.

\section{La Constitución ecológica}

El Constituyente de 1991 instituyó nuevos parámetros en la relación persona y naturaleza. Concedió una importancia cardinal al medio ambiente que ha llevado a catalogarla como una "Constitución ecológica" o "Constitución verde" Así lo sostuvo la Corte en la sentencia C-126 de 1998:

La Constitución de 1991 modificó profundamente la relación normativade la sociedad colombiana con la naturaleza. Por ello esta Corporación haseñalado (...) que la protección del medio ambiente ocupa un lugar tantrascendental en el ordenamiento jurídico que la Carta contiene una verdadera "Constitución ecológica", conformada por todas aquellas disposiciones que regulan la relación de la sociedad con la naturaleza y que buscan proteger el medio ambiente.

Numerosas cláusulas constitucionales reconocen al medio ambiente un interés superior (...) Este conjunto de disposiciones permiten mostrar la trascendencia que tiene el medio ambiente sano y el vínculo de interdependencia con los seres humanos y no humanos. 


\section{El bien jurídico constitucional del medio ambiente y los deberes correlativos}

La Constitución ecológica lleva implícita el reconocimiento al medio ambiente de una triple dimensión: De un lado, es un principio que irradia todo el orden jurídico puesto que es obligación del Estado proteger las riquezas naturales de la Nación. De otro lado, aparece como el derecho de todas las personas a gozar de un ambiente sano, derecho constitucional que es exigible por diversas vías judiciales. Y, finalmente, de la Constitución ecológica derivan un conjunto de obligaciones impuestas a las autoridades y a los particulares. Es más, en varias oportunidades este Tribunal ha insistido en que "la importancia del medio ambiente en la Constitución es de tal magnitud que implica para el Estado "unos deberes calificados de protección" (Corte Constitucional, T-5.603.544, 2017).

La defensa del medio ambiente constituye un objetivo de principio dentro de la forma organizativa de Estado social de derecho acogida en Colombia. Ha dicho la Corte que constitucionalmente:

(...) involucra aspectos relacionados con el manejo, uso, aprovechamiento y conservación de los recursos naturales, el equilibrio de los ecosistemas, la protección de la diversidad biológica y cultural, el desarrollo sostenible, y la calidad de vida del hombre, entendido como parte integrante de ese mundonatural, temas, que, entre otros, han sido reconocidos ampliamente por nuestra Constitución Política en muchas normas que establecen claros mecanismos para proteger este derecho y exhortan a las autoridades a diseñar estrategias para su garantía y su desarrollo. En efecto, la protección del medio ambiente ha adquirido en nuestra Constitución un carácter de objetivo social, que al estar relacionado adicionalmente con la prestación eficiente de los servicios públicos, la salubridad y los recursos naturales como garantía de la supervivencia de las generaciones presentes y futuras, ha sido entendido como una prioridad dentro de los fines del Estado y como un reconocimiento al deber de mejorar la calidad de vida de los ciudadanos (Corte Constitucional T-160038, 1998). 
Dados los factores perturbadores y el riesgo que enfrenta el medio ambiente que le ocasionan daños irreparables e inciden nefastamente en la existencia de la humanidad, la Corte ha sostenido el carácter de derecho fundamental por conexidad, al resultar ligado indefectiblemente con los derechos individuales a la vida y a la salud de las personas. Su reconocimiento general en el ordenamiento constitucional es como derecho colectivo, cuya vía judicial de protección son las acciones populares (art. 88 superior). El ambiente sano también tiene el carácter de servicio público erigiéndose junto con la salud, la educación y el agua potable, en un objetivo social cuya realización material encuentra pleno fundamento en el fin esencial de propender por el mejoramiento de la calidad de vida de la población del país. (Artículos 2, 49, 365 y 366 superiores). Es claro, entonces, que el Estado colombiano frente a las actividades mineras se muestra negligente en la protección de los derechos fundamentales de la población de Aracataca al paso del tren carbonero por el casco urbano de su municipio.

El tercer tema de discusión propuesto por la investigación de Arias y Tapia (2018) está relacionado con la naturaleza del movimiento de resistencia social de la población de Aracataca al paso del tren carbonero por su municipio, el cual desde la investigación se considera que con su accionar se inscribe en la tradición:

(...) de innumerables movimientos ambientalistas y movimientos indígenas desarrollados en la región latinoamericana, con quienes comparten los ideales de protección al medioambiente, los territorios y la salud humana. Juntos se oponen no solamente al sistema capitalista sino, más ampliamente, a la implementación de las políticas neoliberales en los países latinos (Arias y Tapia, pág. 78).

Pero también se identifica con las especificidades de las dinámicas de resistencia de las comunidades a la gran minería en el caribe colombiano, especialmente en los departamentos de la Guajira, Cesar y Magdalena, donde los conflictos socio-ambientales han estado presentes en toda la ruta del carbón, es decir, en el momento de la explotación, del transporte y del embarque. Efectivamente, arriba se señaló que en cada uno de los momentos de la ruta del carbón existían afectaciones socio-ambientales, pero que, a su vez, en cada uno de ellos éstas tenían sus propias especificidades. En ese sentido, las afectaciones socio-ambientales de la población de Aracataca son 
por el paso del tren carbonero por el casco urbano del municipio (y, entre otros, la construcción de una segunda línea férrea y un muro separador), las cuales han sido enfrentadas con vehemencia y de manera colectiva desde el principio, tal como Giraldo (2010) lo muestra a través de la nota periodística del El Informador de Santa Marta en el 2006:

Desde el alcalde de Aracataca, Pedro J. Sánchez; pasando por las distintas autoridades locales, empresarios, dirigentes políticos, docentes, estudiantes y comunidad en general, marchó ayer con relación a la construcción de una segunda línea férrea, la cual según expresaron dividiría al municipio. (...) Sánchez Rueda dijo que hacer una segunda línea férrea en el municipio de Aracataca 'dividiría el pueblo en dos y creo que como administración municipal tengo que unirme al clamor de la comunidad' (El Informador, 23 de agosto de 2006). Ahora bien, después de casi dos décadas de lucha del movimiento de resistencia social de la población de Aracataca al paso de tren carbonero por el casco urbano de su municipio, afrontando las preferencias del Estado con las multinacionales mineras, el movimiento también ha tenido que aguantar la presencia de los "paraestados", tal como lo señaló el investigador Fabio Silva Vallejo en una entrevista que le fue realizada en Santa Marta en 2017.

Hay un elemento que atraviesa los movimientos sociales y es la persecución política a través de los Paraestados, que quiere decir eso, el movimiento social cambia su razón de ser y más que su razón de ser cambia su estructura y se construye se otro tipo movimiento social, primero que va en silencio; los movimientos sociales no son silencio, como lo diría Scott porque el movimiento social es algo que va usted por la calle porque no hay peligro de que venga un tercer actor (Paraestado y lo silencie) no, pero aquí sí y los medios de comunicación se convirtieron en instrumento del Paraestado y no permitieron que se convirtiera el movimiento en instrumento de denuncia, eso que quiere decir, que las formas que se dan constantemente para el caribe colombiano están atravesadas por ese Paraestado, entonces obliga a que la protesta social cambie de forma, dependiendo de la fuerza de los Paraestados y las formas de resistencia son diferentes, es decir, Fundación, Aracataca, todo este eje bananero ha sido históricamente una zona de Paraestados, porque toda esta es una zona que la hacen producir y se han apoderado de eso las familias aristocráticas del Magdalena Grande y esas familias aristocráticas siempre han tenido una 
relación estrecha con los Paraestados y por supuesto como es un Paraestado delegan como todo Estado un sistema de vigilancia.

El Estado colombiano produce una policía para vigilar, para que estemos supuestamente bien, los Paraestados construyen lo mismo, entonces construyen un sistema de vigilancia, dependiendo de la cantidad y esas manifestaciones se dan, es decir, esas manifestaciones ahí si hay que mirarlas desde Scott, desde las formas ocultas porque no solamente son formas que se dan por los periódicos porque los periódicos no lo manifiestan, eso se puede ver en los registros de las masacres que se dan por esa zona. Pero, no obstante lo arriba señalado, se pueden considerar como logros parciales del Movimiento de Resistencia Social de la Población de Aracataca a las siguientes acciones colectivas:

- Convocar a las instituciones públicas, privadas y comunidad para un mismo propósito: la resistencia al paso del tren carbonero por el casco urbano del municipio

- La crear un Comité Pro-defensa de la calidad de vida con representación de los pobladores de Aracataca

- Constituir una veeduría ciudadana pro-defensa de Aracataca (VEECPRODEA)

- La creación de una mesa de diálogo con Fenoco, cuyo eje principal de discusión ha sido la suspensión de la segunda vía férrea.

- Visibilizar la problemática ante la Corte Constitucional, la cual:

Mediante la Sentencia 662 de 2014 exhorta al gobierno nacional que retome el Auto 2952 ratificado por Auto 694 de 2009, para que de una vez por todas corrija lo daños causados no solo a Aracataca, sino también a Bosconia, Zona Bananera y Fundación; situación que el gobierno nacional ha hecho caso omiso puesto que no ha atendido la exhortación que le hace la máxima instancia judicial del país como lo es la Corte Constitucional (...) (Mora, 2017) Entrevista a Alejandro Mora Suárez Concejal del municipio de Aracataca 


\section{Conclusiones}

En primer lugar, que la mayoría de los estudios sobre la minería muestran que cuando se hace un análisis integral de la implementación del modelo extractivista en Colombia el resultado se muestra negativo, aún en lo económico, que es lo más defendido por el Estado, puesto que sobre los impactos ambiental, social, cultural, territorial y de salud humana, es evidente su negatividad.

En segundo lugar, que los conflictos socio-ambientales generados por el modelo extractivista ha develado una conflictividad ínter-institucional entre el poder local y el poder central del Estado, por la protección de los derechos fundamentales de los ciudadanos, pues el Estado central interpreta amañadamente la jerarquía jurídica de los derechos establecidos en la Constitución Política de 1991.

En tercer lugar, que la población de Aracataca ha desarrollado por casi dos décadas un movimiento social de resistencia al paso de tren carbonero por el casco urbano del municipio, con lo cual este movimiento se ha inscrito en la tradición de resistencia al extractivismo en Latinoamérica, pero también con la dinámicas de resistencia de las comunidades a la gran minería en el Caribe colombiano, especialmente en los Departamentos de la Guajira, Cesar y Magdalena, donde los conflictos socio-ambientales han estado presentes en toda la ruta del carbón, es decir, en los momentos de la explotación, del transporte y del embarque.

\section{Referencias}

Beltrán, Ternera y Giraldo (2010). Las paradojas de una bonanza: Impactos de la actividad carbonera en los departamentos del Cesar y Magdalena. Santa Marta. Colciencias

Corte Constitucional (31 de agosto de 1998) Sentencia T-160038

Corte Constitucional (15 de mayo de 2017) Sentencia T-5603544

Espitia Zamora, J. E. (2014). La distribución regional de la actividad agrícola, minera y no minera en Colombia 1975-2012 (Minería en Colombia, págs.381-446) Contraloría General de la República. Bogotá. 
Garavito, L... (2016). Crecimiento Económico, Empleo y Desarrollo en Colombia (En Minería y Desarrollo, pp. 177-209). Externado de Colombia. Bogotá.

Garay Salamanca, L. J. (2013). Minería en Colombia. Contraloría General de la República. 3 Tomos.

Ghon, M. D. (1997). Teorías dos movimentos sociasis. Paradigmas Classicos. E. Sao Paulo: Edico Loyola.

Giraldo Barbosa, J. E. (2010). Sistema de transporte del carbón: impactos y la evocación frustrada por el choque de trenes (En Las paradojas de una bonanza: Impactos de la actividad carbonera en los departamentos del Cesar y Magdalena, págs. 90-139). Santa Marta, Universidad del Magdalena-Colciencias.

Llinás Vargas, M. (2010). Cómo digerir el “boom” minero-energético. Portafolio. Bogotá.

Negrete Montes, R. E. (2013). Consulta previa: consideraciones constitucionales-perspectiva nacional (En Minería en Colombia, págs.281335). Contraloría General de la República. Bogotá.

Olivero Verbel, J. y Otros (2013). Implicaciones de la minería del carbón en el ambiente y la salud humana: una aproximación abreviada al estado del arte (En Minería en Colombia, págs. 229-242). Contraloría General de la República. Bogotá.

Pardo, L. Á. (2014), Una política integral minera desde la perspectiva de un sistema complejo: hacia un modelo alternativo (En Minería en Colombia, págs.31-77) Contraloría General de la República. Bogotá.

Rudas Lleras, G. (2013). La paradoja de la minería y el desarrollo: análisis departamental y municipal para el caso de Colombia (En Minería en Colombia, págs. 28-84). Contraloría General de la República. Bogotá. 